\title{
The Use of Barbed Suture for Vaginal Cuff Closure in Total Laparoscopic Hysterectomy
}

\section{Abstract}

Objective: The aim of this work is to evaluate the safety and efficacy of the barbed unidirectional suture in a total laparoscopic hysterectomy.

Materials and Methods: A retrospective and descriptive study was conducted, which included all of the patients that have undergone laparoscopic hysterectomy and closure of the vaginal cuff, using barbed sutures (V-loc ${ }^{\mathrm{TM}} 90$ Device, CovidienTM), during the period between May 2011 and December 2014. We have analyzed the general characteristics, indications, history of previous surgery and the presence of fever or surgical re-intervention due to pelvic abscesses. The appearance of a vaginal cuff hematoma, active bleeding and vaginal cuff dehiscence were also taken into account.

Results: A hundred and twenty-one laparoscopic hysterectomies with vaginal cuff closure, using barbed sutures, were performed. There was a report of one case of bladder lesion and another case of ureter lesion. Of all patients, four $(3.3 \%)$ of them presented fever while one $(0.8 \%)$ presented paralytic ileus. Three $(2.4 \%)$ patients suffered active vaginal bleeding, and one presented a partial dehiscence of the vaginal cuff. And finally, two $(1.6 \%)$ cases of vaginal cuff hematomas were observed and in one $(0.8 \%)$ of the cases, a pelvic abscess was detected.

Conclusion: The use of a unidirectional barbed suture is a safe technique for vaginal cuff closure in laparoscopic total hysterectomies.

Keywords: Barbed suture; Laparoscopic hysterectomy; Laparoscopic suturing; Vaginal cuff closure

\section{Introduction}

The Hysterectomy procedure is the most frequently performed gynecological surgery in the world [1]. This procedure can be carried out using an abdominal, vaginal, and laparoscopic approach. The percentage of hysterectomies performed via laparoscopy varies. For instance, $12 \%$ of the hysterectomies performed in the USA are performed laparoscopically [2], while $15 \%$ of the hysterectomies in the UK are performed via laparoscopy [3]. In our center, at the Castellon General University Hospital, the percentage of laparoscopic hysterectomies is $34 \%$. In the scientific literature, the rate of complications of vaginal cuff closure post laparoscopic hysterectomy ranges from 0 to $5 \%[4,5]$, the most common complications being: vaginal bleeding, dehiscence of the vaginal cuff and infection. Numerous strategies were undertaken in order to decrease such complications [6,7]. One solution has been the introduction of the unidirectional barbed suture for vaginal cuff closure. The latter has demonstrated a lower technical difficulty [8-10] as well as a decrease in the surgical time, intraoperative complications and a lower incidence of suture dehiscence $[9,11]$. The use of the barbed suture has been described in multiple gynecological procedures, such as the hysterectomy, myomectomy and colposacropexy [1214]. The favorable results obtained in several studies suggest that the suture material has the potential to become an asset in gynecological surgery. The aim of this work is to evaluate the safety and efficacy of the barbed unidirectional suture in a total laparoscopic hysterectomy.

\section{Materials and Methods}

A retrospective and descriptive study was conducted at the Castellon General University Hospital, which included all of the patients that have undergone laparoscopic hysterectomy and closure of the vaginal cuff, using barbed sutures (V-loc ${ }^{\mathrm{TM}}$ 90Device, CovidienTM), during the period between May 2011 and December 2014. Our surgical protocol for laparoscopic hysterectomy includes a pre-surgical phase, where the patient receives mechanical bower preparation as well as thromboprofilaxis using low molecular weight heparin. Two grams of amoxicillin-clavulanic acid are administered intravenously, as antibiotic prophylaxis, during the induction of anesthesia. We generally use the Clermont-Ferrand uterine manipulator. As for the laparoscopic technique, a $12 \mathrm{~mm}$ Hasson trocar is introduced, followed by three accessory ports: a $10 \mathrm{~mm}$ suprapubic trocar y two $5 \mathrm{~mm}$ trocars in both iliac fossa. After carrying out the laparoscopic hysterectomy according to our surgical protocol, the colpotomy is performed, using the coagulation of the monopolar electrode at a power setting of $40 \mathrm{~W}$, pressing against the colpotomizer of the uterine manipulator. The surgical specimen is extracted through the vagina. Vaginal cuff hemostasis is ensured using the bipolar forceps, if necessary, at a power setting of $30 \mathrm{~W}$.

We generally use $3 / 0$ barbed monofilament suture(V-loc ${ }^{\mathrm{TM}} 90$ Device, CovidienTM)or $2 / 0$ vicryl suture for the vaginal cuff closure. The vaginal cuff may be left unsutured in very few cases. We usually perform a transverse closure of the vaginal cuff, always initiating the suturing process at the right angle of the vagina, fixating the uterosacral ligaments to the vaginal cuff. All the cases 
presented here were conducted or supervised by the main author (JLH). Both demographic and surgical variables were recorded in every patient file. The demographic variables included: age, body mass index (BMI), parity, surgical indication and the history of previous surgeries. As for the surgical variables, the following were documented: Surgery duration, blood loss, intraoperative complications, the presence of fever, hospitalization days and hospital readmission or surgical re-intervention due to pelvic abscesses. The appearance of a vaginal cuff hematoma, active bleeding and vaginal cuff dehiscence were also taken into account.

\section{Results}

During the period between May 2011 and December 2014, a hundred and fifty-seven laparoscopic hysterectomies were performed in the Castellon University General Hospital. The vaginal cuff was left open and unsutured in $5(3.2 \%)$ patients, 31 $(19,7 \%)$ vaginal cuffs were closed using Vicryl, and 121 (77.1\%) cuff closures were performed using reabsorbible barbed suture(V$\operatorname{loc}^{\mathrm{TM}} 90$ Device, CovidienTM). In our study we have only taken into account the patients who have had their vaginal cuff closed with barbed suture. The demographic characteristics of the patients are summarized in Table 1.

Table 1: General Characteristics of the Patients Studied.

\begin{tabular}{|c|c|}
\hline Characteristics & Average or Percentage \\
\hline BMI & $27.6(18.6-40)$ \\
\hline Age & $54.08(23-94)$ \\
\hline \multicolumn{2}{|c|}{ Previous Surgeries } \\
\hline Cesarean & $14.10-5)$ \\
\hline Vaginal plastias & $2.7 \%$ \\
\hline Adnexectomy & $6 \%$ \\
\hline Conization & $2.5 \%$ \\
\hline Myomectomy & $1.7 \%$ \\
\hline Appendicectomy & $4.1 \%$ \\
\hline \multicolumn{2}{|c|}{ Surgical Indication } \\
\hline Myoma & $30.8 \%$ \\
\hline Hypermenorrea & $5.0 \%$ \\
\hline Hyperplasia without Atypia & $0.8 \%$ \\
\hline Hyperplasia with Atypia & $5.8 \%$ \\
\hline Cervical Cancer & $7.5 \%$ \\
\hline Endometrial Cancer & $35.0 \%$ \\
\hline Ovarian Cancer & $8.3 \%$ \\
\hline Endometriosis & $4.2 \%$ \\
\hline Others & $2.5 \%$ \\
\hline
\end{tabular}

The average surgery duration was 112.08 minutes (60-240), with an estimated hematic loss of $269 \mathrm{ml}$ (50-600). The average number of days of hospitalization was 3.75 days (2-10). As for the intraoperative complications, we report one case of bladder lesion $(0.8 \%)$ and another case of ureter lesion $(0,8 \%)$, while there were no cases on intestinal lesion. Of all patients, four (3.3\%) presented fever while one $(0.8 \%)$ presented aparalytic ileus during the postoperative period. Three $(2.4 \%)$ patients suffered active vaginal bleeding, and one $(0.8 \%)$ presented a partial dehiscence of the vaginal cuff. And finally, two (1.6\%) cases of vaginal cuff hematomas were observed and in one $(0.8 \%)$ of the cases, a pelvic abscess was detected. Of the previously mentioned complications, only one patient has needed reintervention due to a vaginal cuff hematoma, which was resolved by laparoscopy. During the postoperative period, nine patients visited the emergency room, and their chief complaint included: vaginal bleeding (22\%), fever (11\%), abdominal pain (22\%), abdominal distention (11\%), and urinary symptoms $(11 \%)$.

\section{Discussion}

Our surgical team has adopted the use of new techniques and materials to ensure a minimally invasive approach in gynecological surgery. One such technique is the use of the barbed suture for vaginal cuff closure during laparoscopic hysterectomies. This asset is used in our center since 2011, which allows us to analyze its results.

The use of barbed suture for the vaginal cuff closure is technically easier to achieve, compared to vaginal cuff closure using Vicryl. The barbed suture allows a better distribution of the tension throughout the suture and, additionally, does not require knots. This allows us to reduce the surgical time in the last step of the laparoscopic hysterectomy. This can be a helpful tool for the surgeon, especially in long interventions in which the closure of the vaginal cuff is performed when the latter is already exhausted, thus avoiding possible complications. As for the surgical duration, various scientific research papers presented better results associated with barbed suture compared to Vicryl or other types of sutures [1]. In our case, we cannot affirm that there is a notable reduction in surgical duration, as this is not a comparative study; however, our results are similar to those published in other series $[1,9]$.

The barbed monofilament suture has a similar bacterial adherence as the standard monofilament, but has a lower bacterial adherence compared to other types of suture material [15]. We only report a case of vaginal cuff abscess in our series, which was resolved satisfactorily by draining it, by interventional radiology, and antibiotherapy.

The dehiscence of the vaginal cuff is a complication that occurs most frequently after a total laparoscopic hysterectomy, and is less likely to happen after abdominal or vaginal hysterectomy. Such an adverse effect mainly depends on the closing technique used on the vaginal cuff $[16,17]$, the suture material used $[17,18]$, and finally, the type of energy used for the colpotomy and the hemostasis of the vaginal cuff [19-22]. Blikkendaal [7] published a retrospective study in which he describes the incidence of vaginal cuff dehiscence in patients after a total laparoscopic hysterectomy. He also compares the vaginal cuff closure transvaginally with a laparoscopic approach, using Vicryl interrupted sutures and continuous barbed sutures. The same author describes a vaginal cuff dehiscence rate of $1.3 \%$ in the first group, 3.3\% in the second one, and $2.4 \%$ in the third group. The incidence rate of dehiscence in our case is $0.8 \%$.

Regarding the influence of the energy used for the colpotomy on the incidence of vaginal cuff dehiscence, Gruber [21] describes a major tissue lesion with a possible increase in dehiscence when bipolar energy is used. We generally use monopolar energy for the colpotomy, as is indicated by our protocol, and we reserve the 
use of bipolar energy only for arterial bleedings. A hemorrhage originating from a venous territory is generally controlled using sutures. This recommendation is based on the low incidence of dehiscence reported after abdominal hysterectomies, in which the vaginal cuff is sutured normally without having to use energy for hemostasis [23].

Studies with a greater number of patients, comparing the use of different techniques and suture materials, are needed to affirm that the laparoscopic closure of the vaginal cuff using barbed sutures is superior to the others previously described.

\section{Conclusion}

This study has demonstrated that the unidirectional monofilament barbed suture(V-loc ${ }^{\mathrm{TM}} 90$ Device, CovidienTM) can be safely used and is effective for vaginal cuff closure after having performed total laparoscopic hysterectomies. In our experience, we believe that this technique should be considered and included as one of the steps of the laparoscopic total hysterectomy.

\section{References}

1. Medina BC, Giraldo CH, Riaño G, Hoyos LR, Otalora C (2014) Barbed suture por vaginal cuff closure in laparoscopic hysterectomy. JSLS 18(1): 83-88.

2. Wu JM, Wechter ME, Geller EJ, Nguyen TV, Visco AG (2007) Hysterectomy rates in the United States, 2003. Obstet Gynecol 110(5): 1091-1095.

3. Sokol AI, Green IC (2009) Laparoscopic hysterectomy. Clin Obstet Gynecol 52(3): 304-312.

4. Argüello R, Hoyos EA, Perdom PW (2003) Total laparoscopic hysterectomy in 202 patients (in Spanish). Rev Colomb Obstet Ginecol 54(4): 258-264.

5. O’Hanlan KA, Dibble SL, Garnier AC, Reuland ML (2007) Total laparoscopic hysterectomy: technique and complications of 830 cases. JSLS 11(1): 45-53.

6. Colombo M, Maggioni K, Zanini A, Rangoni G, Scalambino S, et al (1995) A randomized trial of open versus closed vaginal vault in the prevention of postoperative morbidity after abdominal hysterectomy. Am J Obstet Gynecol 173(6): 1807-1811.

7. Blikkendaal MD, Twijnstra AR, Pacquee SC, Rhemrev JP, Smeets MJ, et al. (2012) Vaginal cuff dehiscence in laparoscopic hysterectomy: influence of various suturing methods of the vaginal vault. Gynecol Surg 9(4): 393-400.

8. Greenberg JA, Einarsson JI (2008) The use of bidirectional barbed suture in laparoscopic myomectomy and total laparoscopic hysterectomy. J Minim Invasive Gynecol 15(5):621-623.

9. Einarsson JI, Vellinga TT, Twijnstra AR, Chavan NR, Suzuki Y, et al. (2010) Bidirectional barbed suture: an evaluation of safety and clinical outcomes. JSLS 14(3): 381-385.
10. Alessandri F, RemorgidaV,Venturini PL, Ferrero S(2010) Unidirectional barbed suture versus continuous suture with intracorporeal knots in laparoscopic myomectomy: a randomized study. J Minim Invasive Gynecol 17(6): 725-729.

11. Bogliolo S, Nadalini C, Iacobone AD, Valentina Musacchi, Carus AP (2013) Vaginal cuff closure with absorbable bidirectional barbed suture during total laparoscopic hysterectomy. Eur J of Obstet Gynecol Reprod Biol 170(1): 219-221.

12. Angioli R, Plotti F, Montera R, Damiani P, Terranova C, et al. (2012) A new type of absorbable barbed suture for use in laparoscopic myomectomy. Int J Gynaecol Obstet 117(3): 220-223.

13. Einarsson JI, Chavan NR, Suzuki Y, Jonsdottir G, Vellinga TT, et al. (2011) Use of bidirectional barbed suture in laparoscopic myomectomy: evaluation of perioperative outcomes, safety, and efficacy. J Minim Invasive Gynecol 18(1): 92-95.

14. Herraiz JL, Llueca JA, Catala C, Oliva C, Serra A, et al. (2015) Colposacropexia laparoscópica con malla y anclaje de titanio para la corrección del prolapso de cúpula vaginal. Prog Obstet Ginecol 58(5): 246-251.

15. Fowler JR, Perkins TA, Buttaro B, Truant A (2013) Bacteria Adhere Less to Barbed Monofilament Than Braided Sutures in a Contaminated Wound Model. Clin Orthop Relat Res 471(2): 665-671.

16. Uccella S, Ceccaroni M, Cromi A, Malzoni M, Berretta R, et al. (2012) Vaginal cuff dehiscence in a series of 12,398 hysterectomies: effect of different types of colpotomy and vaginal closure. Obstet Gynecol $120(3): 516-523$

17. Hur HC, Guido RS, Mansuria SM, Hacker MR, Sanfilippo JS, et al. (2007) Incidence and patient characteristics of vaginal cuff dehiscence after different modes of hysterectomies. J Minim Invasive Gynecol 14(3): 311-317.

18. Yuce K, Dursun P, Gultekin M (2005) Post hysterectomy intestinal prolapse after coitus and vaginal repair. Arch Gynecol Obstet 272(1): 80-81.

19. Jeung IC, Baek JM, Park EK, Lee HN, Kim CJ, et al. (2010) A prospective comparison of vaginal stump suturing techniques during total laparoscopic hysterectomy. Arch Gynecol Obstet 282(6): 631-638.

20. Nezhat CH, Nezhat F, Seidman DS, Nezhat C (1996) Vaginal vault evisceration after total laparoscopic hysterectomy. Obstet Gynecol 87(5 Pt 2): 868-870.

21. Gruber DD, Warner WB, Lombardini ED, Zahn CM, Buller JL (2011) Laparoscopic hysterectomy using various energy sources in swine: a histopathologic assessment. Am J Obstet Gynecol 205(5): 494-496.

22. Walsh CA, Sherwin JR, Slack M (2007) Vaginal evisceration following total laparoscopic hysterectomy: case report and review of the literature. Aust N Z J Obstet Gynaecol 47(6): 516-519.

23. Thompson JD, Warschaw J (1997) Hysterectomy. In: Rock JA, Thompson JD (Eds.) Te Linde's operative gynecology, $\left(8^{\text {th }}\right.$ edn), Lippincott, Philadelphia, USA, pp. 771-854. 